\title{
3D Energy Absorption Diagram Construction of Paper Honeycomb Sandwich Panel
}

\author{
Dongmei Wang $\mathbb{D}^{1},{ }^{1}$ Ziyou Bai, ${ }^{1}$ and Qianghua Liao ${ }^{2}$ \\ ${ }^{1}$ School of Media and Communication, Shenzhen Polytechnic, Shenzhen 518055, China \\ ${ }^{2}$ School of Mechanical and Electrical Engineering, Shenzhen Polytechnic, Shenzhen 518055, China \\ Correspondence should be addressed to Dongmei Wang; 394794521@qq.com
}

Received 12 September 2017; Revised 5 January 2018; Accepted 10 January 2018; Published 7 March 2018

Academic Editor: Francesco Franco

Copyright (c) 2018 Dongmei Wang et al. This is an open access article distributed under the Creative Commons Attribution License, which permits unrestricted use, distribution, and reproduction in any medium, provided the original work is properly cited.

\begin{abstract}
Paper honeycomb sandwich panel is an environment-sensitive material. Its cushioning property is closely related to its structural factors, the temperature and humidity, random shocks, and vibration events in the logistics environment. In order to visually characterize the cushioning property of paper honeycomb sandwich panel in different logistics conditions, the energy absorption equation of per unit volume of paper honeycomb sandwich panel was constructed by piecewise function. The three-dimensional (3D) energy absorption diagram of paper honeycomb sandwich panel was constructed by connecting the inflexion of energy absorption curve. It takes into account the temperature, humidity, strain rate, and characteristics of the honeycomb structure. On the one hand, this diagram breaks through the limitation of the static compression curve of paper honeycomb sandwich panel, which depends on the test specimen and is applicable only to the standard condition. On the other hand, it breaks through the limitation of the conventional 2D energy absorption diagram which has less information. Elastic modulus was used to normalize the plateau stress and energy absorption per unit volume. This makes the $3 \mathrm{D}$ energy absorption diagram universal for different material sandwich panels. It provides a new theoretical basis for packaging optimized design.
\end{abstract}

\section{Introduction}

Paper honeycomb sandwich panel is an environmentalfriendly, low-cost, good-mechanical property material, which is widely used as cushioning material for packaging for large mechanical and electrical products in recent years. It has been highly concerned by the packaging industry. Paper honeycomb sandwich panel has also been successfully used in furniture and building industry as loading and filling material. In short, it has broad market prospects.

Nowadays, the research of the cushioning property of paper honeycomb sandwich panel is mostly focused on a specific configuration of honeycomb. Therefore, the results are dependent on the test samples and are limited in the practical application. Xin and Guo [1], Cai and $\mathrm{Lu}$ [2], and Zhang [3] studied the influence of temperature and relative humidity on cushioning property and static compressive property of paper honeycomb sandwich panel and found that as the relative humidity increased and the temperature decreased, the average critical stress, plateau stress, and the cushioning property of paper honeycomb sandwich panel declined. Cui and Chen [4] studied the effects of different levels of precompression on the cushioning performance of paper honeycomb sandwich panel. The results showed that with the increase in the precompression level, the static compression performance, static cushioning performance, and energy absorption of paper honeycomb sandwich panel reduced accordingly. The above researches were based on tests, and the simple qualitative relationship between the cushioning property of paper honeycomb sandwich panel and some parameters was obtained.

Combined with the theory, the cushioning property of paper honeycomb sandwich panel was studied from the structure by some scholars. Wang et al. $[5,6]$ assessed the critical stress of paper honeycomb sandwich panel and obtained the assessment equation of critical stress. Then they established a static compression model of paper honeycomb sandwich panel, which is only suitable for the assessment 
of the mechanical properties of paper honeycomb sandwich panel under the static loading condition. In practice, cushion curve is used to estimate the cushioning property of material [7]. Based on the cushion curve, Maiti et al. [8] proposed the energy absorption diagram which can be constructed according to the compressive stress-strain curve of materials. The relationship between the stress and energy absorption of cushion material can be characterized by energy absorption diagram. In recent years, some domestic scholars studied the characterization methods of energy absorption of paper honeycomb sandwich panel actively. Wang et al. [9] and Wang and $\mathrm{Lu}[10]$ constructed the energy absorption curve of per unit volume paper honeycomb sandwich panel, and then they linked the optimal inflexion point of energy absorption curve to build the energy absorption diagram of per unit volume paper honeycomb sandwich panel with different structures. Xiong et al. [11] constructed the 3D image of corrugated paper among moisture content, temperature, and relative humidity by experiments and studied the influence of moisture content on elastic modulus of corrugated paper. The results showed that the moisture content decreases with temperature and increases with relative humidity. The elastic modulus decreases with moisture content. Wang and E [12] and E and Wang [13] studied the influence of relative humidity on the cushioning property of paper honeycomb sandwich panel and fitted the relationship equation of energy absorption and various relative humidity. The energy absorption diagram of per unit volume paper honeycomb sandwich panel which considered the relative humidity was drawn. Wang et al. [14] developed mathematical model in which the structure factors and temperature and relative humidity are concerned through the cooperation of theories and experiments. And the model is normalized by the elastic modulus of the medium under controlled condition; thus this model can be used to predict the effect of various temperature and relative humidity on the plateau stress of multilayer corrugated sandwich structure under flatwise compression.

On the basis of a great deal of research on static compression, scholars have further studied the energy-absorbing properties of the paper honeycomb sandwich panel under dynamic impact. E and Wang [15] investigated the effect of the strain rate on the mechanical behavior and energy absorption of paper honeycomb by experiments. The experimental results show that the load-carrying capacity and the energy absorption performance of paper honeycomb are insensitive to the loading speed in low strain rate range $\left(10^{-4}-10^{-2} \mathrm{~s}^{-1}\right)$. Wang and Bai [16] found that there exists a certain strain rate effect which leads to the difference of mechanical properties between dynamic and static condition. A mechanical model was given to estimate plateau stress and yield stress at different strain rates based on experimental data by employing the methods of Cowper-Symonds model and piecewise function. And then Bai et al. [17] according to the mechanical model built the energy absorption diagram under different strain rates. Moreover, they have taken serious experiments and the result showed a good agreement with theoretical models.

From present documents, all energy absorption properties were researched on either relative humidity effect or strain rate effect. The energy absorption diagram of material can be modeled by linking the optimal energy absorption point under two dimensions. However, only a single parameter was considered in the two-dimensional energy absorption diagram which limited its application. The purpose of this paper is to construct 3D diagram of paper honeycomb sandwich panel which took structure, strain rate, and moisture content into account. The diagram took the random shock and variety of environment in the logistics condition into account. This will make the optimization design of paper honeycomb sandwich panel more suitable for the actual logistics environment.

\section{The Evaluation of Plateau Stress and Critical Stress Based on the Effect of Strain Rate and Moisture Content}

The influence of temperature and relative humidity on moisture content and cushioning property of paper honeycomb sandwich panel was studied by reference $[18,19]$. Then a model of moisture content and the prediction equations of critical and plateau stress of paper honeycomb sandwich panel are obtained and verified [6]. We have the equations as follows:

$$
\begin{aligned}
& M \\
& =\frac{20.5 \mathrm{RH}}{(1-0.005432 \mathrm{RH})(1-0.005432 \mathrm{RH}+3.32 \mathrm{RH} / T) T},
\end{aligned}
$$

where $M$ is moisture content of paper honeycomb sandwich panel, \%; RH is relative humidity, \%; $\mathrm{T}$ is temperature, ${ }^{\circ} \mathrm{C}$.

$$
\begin{aligned}
\frac{\sigma_{\mathrm{pk}}}{E_{s}^{0}}= & 19.298 e^{-0.0769 M}\left(\frac{t}{l}\right)^{3}, \\
\frac{\sigma_{\mathrm{pl}}}{E_{s}^{0}}= & 0.0991 e^{-0.0769 M} *\left(\frac{t}{l}\right)^{5 / 3} \\
& +0.01067 e^{-0.0769 M}\left(\frac{t}{l}\right)^{2},
\end{aligned}
$$

where $\sigma_{\mathrm{pk}}$ is critical stress of paper honeycomb sandwich panel, MPa; $\sigma_{\mathrm{pl}}$ is plateau stress of paper honeycomb sandwich panel, $\mathrm{MPa} ; E_{s}^{0}$ is elastic modulus of corrugating medium under standard condition, $\mathrm{MPa} ; \sigma_{\mathrm{pk}} / E_{s}^{0}$ is normalized critical stress; $\sigma_{\mathrm{pl}} / E_{s}^{0}$ is normalized plateau stress; $e=2.7183 ; t / l$ is thickness-to-length ratio; $t$ is thickness of corrugating medium, $\mathrm{mm}$; $l$ is length of honeycomb cell, $\mathrm{mm}$.

Combined with the strain rate effect, $[16,17]$ studied the mechanical behavior of paper honeycomb sandwich panel and obtained the prediction equations of dynamic critical and plateau stress. The equations are as follows:

$$
\begin{aligned}
\frac{\sigma_{\mathrm{pk}}^{\prime}}{E_{s}^{0}} & =\frac{1.8089 \times 10^{-4}}{\left(1-\varepsilon_{p k}\right)\left(1+0.28 \varepsilon_{p k}\right)^{2}}+\frac{\sigma_{\mathrm{pk}}}{E_{s}^{0}} \\
\frac{\sigma_{\mathrm{pl}}^{\prime}}{E_{s}^{0}} & =\frac{\sigma_{\mathrm{pl}}}{E_{s}^{0}}\left[1+\left(\frac{\dot{\varepsilon}}{B}\right)^{1 / q}\right],
\end{aligned}
$$


where $\sigma_{\mathrm{pk}}^{\prime}$ is dynamic critical stress of paper honeycomb sandwich panel, MPa; $\sigma_{\mathrm{pl}}^{\prime}$ is dynamic plateau stress of paper honeycomb sandwich panel, $\mathrm{MPa} ; \sigma_{\mathrm{pk}}^{\prime} / E_{s}^{0}$ is normalized dynamic critical stress; $\sigma_{\mathrm{pl}}^{\prime} / E_{s}^{0}$ is normalized dynamic plateau stress; $\varepsilon_{\mathrm{pk}}$ is the strain when stress reaches the yielding stress; $\dot{\varepsilon}$ is strain rate, $\mathrm{s}^{-1} ; B, q$ are parameters.

Substituting (3) into (5), then the evaluation equation of the plateau stress of paper honeycomb sandwich panel for moisture content and strain rate can be represented as

$$
\begin{aligned}
& \frac{\sigma_{\mathrm{pl}}^{\prime}}{E_{s}^{0}}=\left[0.0991 e^{-0.0769 M} *\left(\frac{t}{l}\right)^{5 / 3}\right. \\
& \left.\quad+0.01067 e^{-0.0769 M}\left(\frac{t}{l}\right)^{2}\right]\left[1+\left(\frac{\dot{\varepsilon}}{B}\right)^{1 / q}\right] .
\end{aligned}
$$

According to the static and dynamic impact tests, $B$ and $q$ can be obtained through curve fitting, as follows [16]:

$$
\begin{aligned}
& B=\frac{1528 t}{l}+20.9, \\
& q=0.5125 .
\end{aligned}
$$

Substituting (7) into (6), then (6) can be simplified as follows:

$$
\begin{aligned}
& \frac{\sigma_{\mathrm{pl}}^{\prime}}{E_{s}^{0}}=\left[0.0991 e^{-0.0769 M} *\left(\frac{t}{l}\right)^{5 / 3}+0.01067 e^{-0.0769 M}\right. \\
& \left.*\left(\frac{t}{l}\right)^{2}\right]\left[1+\left(\frac{\dot{\varepsilon}}{1528 t / l+20.9}\right)^{1.9512}\right] .
\end{aligned}
$$

Substituting $t / l=0.03125, t / l=0.0333$ and $t / l=0.0416$ into (8)

$$
\begin{aligned}
& \frac{\sigma_{\mathrm{pl} 1}^{\prime}}{E_{s}^{0}}=\left(3.0372 e^{-0.0769 M} * 10^{-4}+1.0412 e^{-0.0769 M}\right. \\
& \left.* 10^{-5}\right)\left[1+\left(\frac{\dot{\varepsilon}}{68.25}\right)^{1.9512}\right], \\
& \frac{\sigma_{\mathrm{pl} 2}^{\prime}}{E_{s}^{0}}=\left(3.3772 e^{-0.0769 M} * 10^{-4}+1.1832 e^{-0.0769 M}\right. \\
& \left.* 10^{-5}\right)\left[1+\left(\frac{\dot{\varepsilon}}{71.78}\right)^{1.9512}\right], \\
& \frac{\sigma_{\mathrm{pl} 3}^{\prime}}{E_{s}^{0}}=\left(4.8973 e^{-0.0769 M} * 10^{-4}+1.8465 e^{-0.0769 M}\right. \\
& \left.* 10^{-5}\right)\left[1+\left(\frac{\dot{\varepsilon}}{84.46}\right)^{1.9512}\right] .
\end{aligned}
$$

The 3D diagram was drawn according to (9), as shown in Figure 1.

It can be seen from Figure 1, under the same strain rate conditions, with the increase of moisture content, that the normalized plateau stress exhibited a downward trend, but the decline is small. In the same moisture content conditions, with the increase of strain rate, the stress rose exponentially.

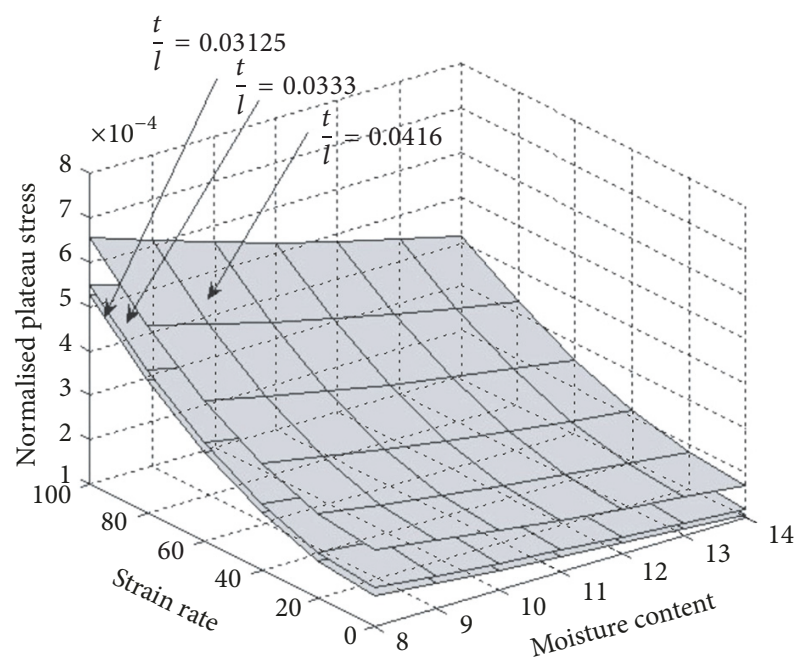

FIGURE 1: 3D diagram of normalized plateau stress of paper honeycomb sandwich panel.

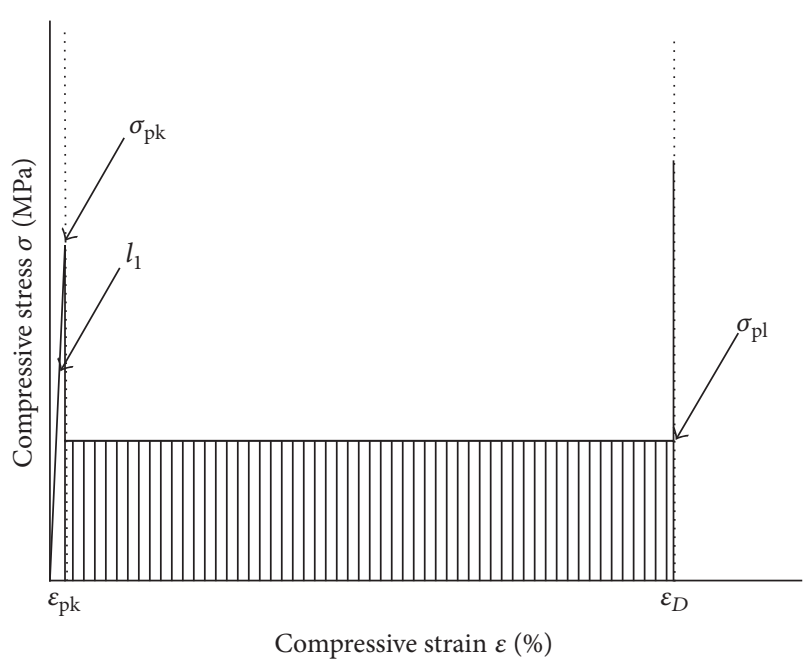

FIgURE 2: Theoretical stress-strain curve of paper honeycomb.

\section{The Evaluation of Energy Absorption Based on the Effect of Strain Rate and Moisture Content}

Figure 2 shows the theoretical stress-strain curve of paper honeycomb sandwich panel. The shaded area in Figure 2 is per unit volume energy absorption theoretical value. The stress-strain curve is divided into three stages: the yield stage, the plateau stage, and densification stage [6]. Integrating $\sigma-\varepsilon$, we can obtain the per unit volume energy absorption value $W$. It is an important factor to measure the energy absorption capacity of paper honeycomb sandwich panel.

The cushioning property of paper honeycomb sandwich panel is affected by various factors, such as the impact from the transport process, environmental temperature, and humidity. The influence of external factors on the cushioning property can be transformed into the influence of moisture content and strain rate. Therefore, the energy absorption 
model is constructed in this paper with taking into account the honeycomb structure, strain rate, and moisture content. This model aims to assess cushioning property of paper honeycomb sandwich panel.

Three stages of dynamic energy absorption equation of paper honeycomb sandwich panel were shown as follows [17, 20]:

$$
\begin{aligned}
& \frac{W}{E_{s}^{0}}=\left(\frac{\sigma^{\prime}}{E_{s}^{0}}\right)^{2} \frac{l \sqrt{3}}{4 t} \quad 0 \leq \varepsilon \leq \varepsilon_{\mathrm{pk}} \\
& \frac{W}{E_{s}^{0}}=\left(\frac{\sigma_{\mathrm{pk}}^{\prime}}{E_{s}^{0}}\right)^{2} \frac{l \sqrt{3}}{4 t}+\frac{\sigma_{\mathrm{pl}}^{\prime}\left(\varepsilon-\varepsilon_{\mathrm{pk}}\right)}{E_{s}^{0}} \quad \varepsilon_{\mathrm{pk}} \leq \varepsilon \leq \varepsilon_{D} \\
& \frac{W}{E_{s}^{0}}=\left(\frac{\sigma_{\mathrm{pk}}^{\prime}}{E_{s}^{0}}\right)^{2} \frac{l \sqrt{3}}{4 t}+\frac{\sigma_{\mathrm{pl}}^{\prime}(1-3.95(t / l))}{E_{s}^{0}} \quad \varepsilon>\varepsilon_{D},
\end{aligned}
$$

where $W$ is the energy absorption per unit volume, J; $W / E_{s}^{0}$ is the normalized energy absorption per unit volume; $\sigma^{\prime}$ is dynamic stress at arbitrary point on stress-strain curve, $\mathrm{MPa}$; $\varepsilon$ is the strain at arbitrary point on stress-strain curve; $\varepsilon_{D}$ is the densification strain.

Substituting (2) into (4) and then substituting (4) and (8) into (10), we can get equations as follows:

$$
\begin{aligned}
& \frac{W}{E_{s}^{0}}=\left(\frac{\sigma^{\prime}}{E_{s}^{0}}\right)^{2} \frac{l \sqrt{3}}{4 t} \quad 0 \leq \varepsilon \leq \varepsilon_{\mathrm{pk}} \\
& \frac{W}{E_{s}^{0}}=\left[\frac{1.8089 \times 10^{-4}}{\left(1-\varepsilon_{\mathrm{pk}}\right)\left(1+0.28 \varepsilon_{\mathrm{pk}}\right)^{2}}\right. \\
& \left.+19.298 e^{-0.0769 M}\left(\frac{t}{l}\right)^{3}\right]^{2} \frac{l \sqrt{3}}{4 t} \\
& +\left[0.0991 e^{-0.0769 M}\left(\frac{t}{l}\right)^{5 / 3}\right. \\
& \left.+0.01067 e^{-0.0769 M}\left(\frac{t}{l}\right)^{2}\right][1 \\
& \left.+\left(\frac{\dot{\varepsilon}}{1528 t / l+20.9}\right)^{1.9512}\right]\left(\varepsilon-\varepsilon_{\mathrm{pk}}\right) \quad \varepsilon_{\mathrm{pk}} \leq \varepsilon \leq \varepsilon_{D} \text {, } \\
& \frac{W}{E_{s}^{0}}=\left[\frac{1.8089 \times 10^{-4}}{\left(1-\varepsilon_{\mathrm{pk}}\right)\left(1+0.28 \varepsilon_{\mathrm{pk}}\right)^{2}}\right. \\
& \left.+19.298 e^{-0.0769 M}\left(\frac{t}{l}\right)^{3}\right]^{2} \frac{l \sqrt{3}}{4 t} \\
& +\left[0.0991 e^{-0.0769 M}\left(\frac{t}{l}\right)^{5 / 3}\right. \\
& \left.+0.01067 e^{-0.0769 M}\left(\frac{t}{l}\right)^{2}\right][1 \\
& \left.+\left(\frac{\dot{\varepsilon}}{1528 t / l+20.9}\right)^{1.9512}\right]\left(1-3.95 \frac{t}{l}\right) \quad \varepsilon>\varepsilon_{D} .
\end{aligned}
$$

Equations (11) are energy absorption model of paper honeycomb sandwich panel. The moisture content and strain rate are taken into account.

In the packaging optimization design of paper honeycomb sandwich panel, the best point is the inflexion which is the terminal point of plateau stage. At this point, namely, when $\varepsilon=\varepsilon_{D}$, the energy absorption value reached the maximum. The energy absorption value of that point consists of two parts which are energy absorption value at the yield stage and plateau stage. The equation was shown as follows:

$$
\frac{W}{E_{s}^{0}}=\left(\frac{\sigma_{\mathrm{pk}}^{\prime}}{E_{s}^{0}}\right)^{2} \frac{l \sqrt{3}}{4 t}+\frac{\sigma_{\mathrm{pl}}^{\prime}(1-3.95(t / l))}{E_{s}^{0}} .
$$

The energy absorption value of yield stage is much less than that of plateau stage. Hence, (12) can be simplified as

$$
\frac{W}{E_{s}^{0}} \approx \frac{\sigma_{\mathrm{pl}}^{\prime}(1-3.95(t / l))}{E_{s}^{0}} .
$$

Substituting (8) into (13), then we can get

$$
\begin{aligned}
\frac{W}{E_{s}^{0}} & =\left[0.0991 e^{-0.0769 M}\left(\frac{t}{l}\right)^{5 / 3}\right. \\
& \left.+0.01067 e^{-0.0769 M}\left(\frac{t}{l}\right)^{2}\right][1 \\
& \left.+\left(\frac{\dot{\varepsilon}}{1528 t / l+20.9}\right)^{1.9512}\right]\left(1-3.95 \frac{t}{l}\right) .
\end{aligned}
$$

Equation (14) is the prediction equation of normalized energy absorption per unit volume of paper honeycomb sandwich panel, which the moisture content and strain rate were concerned with.

Substituting $t / l=0.03125, t / l=0.0333$, and $t / l=$ 0.0416 into (14), the energy absorption of paper honeycomb sandwich panel can be expressed as

$$
\begin{aligned}
& \frac{W_{1}}{E_{s}^{0}}=\left(2.6622 e^{-0.0769 M} * 10^{-4}+9.1267 e^{-0.0769 M}\right. \\
& \left.* 10^{-6}\right)\left[1+\left(\frac{\dot{\varepsilon}}{68.25}\right)^{1.9512}\right], \\
& \frac{W_{2}}{E_{s}^{0}}=\left(2.933 e^{-0.0769 M} * 10^{-4}+1.0275 e^{-0.0769 M}\right. \\
& \left.* 10^{-5}\right)\left[1+\left(\frac{\dot{\varepsilon}}{71.78}\right)^{1.9512}\right] \\
& \frac{W_{3}}{E_{s}^{0}}=\left(4.0925 e^{-0.0769 M} * 10^{-4}+1.5431 e^{-0.0769 M}\right. \\
& \left.* 10^{-5}\right)\left[1+\left(\frac{\dot{\varepsilon}}{84.46}\right)^{1.9512}\right]
\end{aligned}
$$

The $3 \mathrm{D}$ diagram was drawn in accordance with (15), as shown in Figure 3. 
TABLE 1: The comparison of experimental and predictive energy absorption.

\begin{tabular}{lccc}
\hline$W / E_{s}^{0}$ predictive/Experimental $\left(10^{-4}\right)$ & \multicolumn{3}{c}{ Strain rate $\dot{\varepsilon}\left(\mathrm{s}^{-1}\right)$} \\
Thickness-to-length $t / l$ & 10 & 50 & 100 \\
\hline 0.03125 & $1.3060 / 1.4112$ & $1.9638 / 2.0013$ & $3.9350 / 4.1235$ \\
0.0333 & $1.4371 / 1.4532$ & $2.1018 / 2.1429$ & $4.0937 / 4.0125$ \\
0.0416 & $1.9990 / 1.9128$ & $2.6760 / 2.4257$ & $4.7047 / 4.5675$ \\
\hline
\end{tabular}

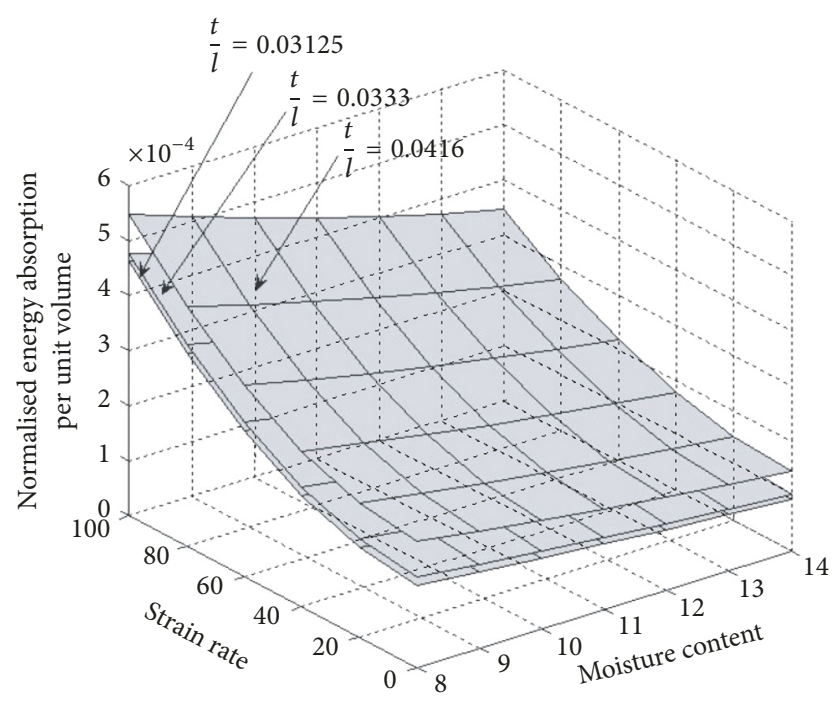

FIGURE 3: 3D diagram of normalized energy absorption of paper honeycomb sandwich panel.

Figure 3 indicates that, in the same moisture content and strain rate conditions, the normalized maximum energy absorption value rises with the increase of $t / l$. At a certain strain rate, the normalized maximum energy absorption value exhibits a downward trend with the increase of moisture content, but the decline range is small. In the same moisture content conditions, the normalized maximum energy absorption value increases with the increase of strain rate. The higher the strain rate is, the more significant the magnitude is.

\section{Experimental Verification}

In the case of moisture content being $10 \%$, (14) can be used to predict the energy absorption of three kinds of paper honeycomb sandwich panel. In order to verify the accuracy of the above equations, three kinds of samples were tested and their energy absorption were obtained. The experimental details and information about geometry and material characteristics are showed in [17]. And then the comparison between the theoretically predictive values and experimental values was shown in Table 1 .

Statistically, the coefficient of determination $R^{2}$ is used in the context of statistical models and is to predict future outcomes on the basis of other related information. It provides a measure of how well future outcomes are likely to be predicted by the model. $R^{2}$ ranges from 0 to 1 . An $R^{2}$

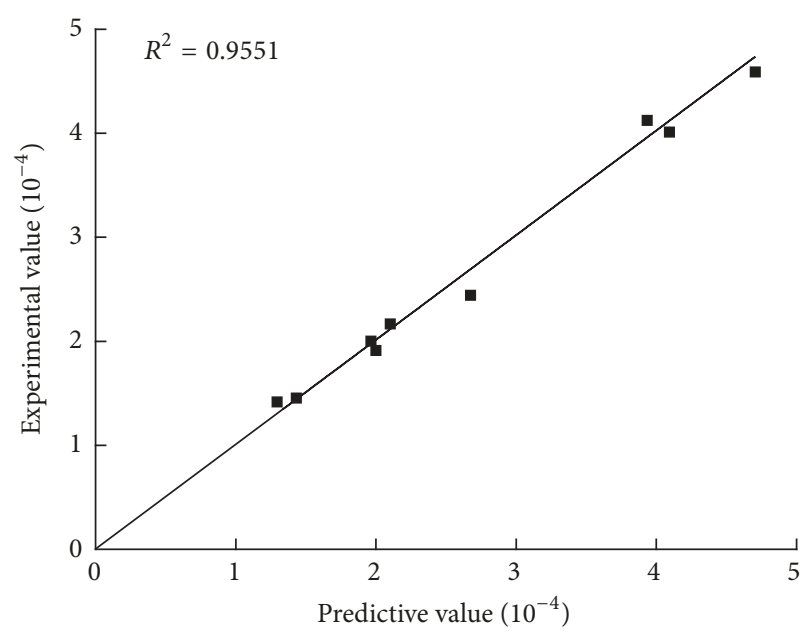

FIgURE 4: The relationship between experimental and predicted energy absorption.

of 1 indicates that the model shows perfect consistency with the experimental data. In Figure $4 R^{2}$ is 0.9551 , which means (14) can be better used to forecast the value of the energy absorption of paper honeycomb sandwich panel.

\section{Conclusion}

In this paper, based on theory and experiment, the energy absorption equation of per unit volume of paper honeycomb sandwich panel was constructed by piecewise function. And then the inflexion of energy absorption curve was connected to construct 3D diagram of energy absorption of paper honeycomb sandwich panel. The diagram can be expressed as the theoretical formula. It takes the temperature, humidity, strain rate, and characteristics of honeycomb structure into account. On the one hand, this diagram breaks through the limitation of the static compression curve of paper honeycomb sandwich panel which depends on the test specimen and is applicable only to standard condition; on the other hand, it breaks through the limitation of the conventional 2D energy absorption diagram which has less information.

Elastic modulus was used to normalize the plateau stress and energy absorption per unit volume, which make the $3 \mathrm{D}$ energy absorption diagram universal for different material sandwich panels. The optimization of paper honeycomb sandwich panel and corrugated sandwich panel can be realized by using $3 \mathrm{D}$ energy absorption diagram, which provides a new theoretical basis for optimum packaging design. In 
the future, once you know the elastic modulus of base paper, with the help of 3D energy absorption diagram, mechanical properties and energy absorption properties of paper honeycomb sandwich plate under different temperature, humidity, and compression state can been predicted without making samples to test.

\section{Conflicts of Interest}

The authors declare that they have no conflicts of interest.

\section{Acknowledgments}

Contract/grant sponsor is National Natural Science Foundation of China, Project (51675349).

\section{References}

[1] C. L. Xin and Y. F. Guo, "Experimental research and analysis on static cushioning properties of honeycomb paperboard," Packaging Engineering, vol. 29, no. 1, pp. 56-58, 2008.

[2] Y. Cai and L. X. Lu, "The influence of humidity on the out-ofplane strength of paper honeycomb," Mechanical and Electrical Information, vol. 17, pp. 41-43, 2004.

[3] Z. K. Zhang, "Experiment research on the effects of relative humidity on the static compression properties of paper honeycomb panel," Packaging Engineering, vol. 29, no. 9, pp. 12-13, 2008.

[4] Y. Cui and L. Chen, "The effect of pre-compression on the energy absorption of honeycomb cardboard," Packaging Engineering, vol. 38, no. 9, pp. 141-145, 2017.

[5] D. M. Wang and Z. W. Wang, "Out-of-plane compressive properties of hexagonal paper honeycombs," Chinese Journal of Mechanical Engineering, vol. 20, no. 2, pp. 115-119, 2007.

[6] D. M. Wang and Q. H. Liao, "Research on the mechanical performance modeling of honeycomb paperboard under quasistatic compression," Packaging Engineering, vol. 27, no. 4, pp. 129-132, 2006.

[7] M. A. Sek, M. Minett, V. Rouillard, and B. Bruscella, "A new method for the determination of cushion curves," Packaging Technology and Science, vol. 13, no. 6, pp. 249-255, 2000.

[8] S. K. Maiti, L. J. Gibson, and M. F. Ashby, "Deformation and energy absorption diagrams for cellular solids," Acta Metallurgica et Materialia, vol. 32, no. 11, pp. 1963-1975, 1984.

[9] D. M. Wang, Z. W. Wang, and Q. H. Liao, "Energy absorption diagrams of paper honeycomb sandwich structures," Packaging Technology and Science, vol. 22, no. 2, pp. 63-67, 2009.

[10] J. Wang and L. X. Lu, "Energy-absorption diagrams of honeycomb paperboards under static compression in different relative humidity," Packaging Engineering, vol. 1, pp. 5-7, 2011.

[11] W. Xiong, J. Wang, and D. M. Wang, "Influence of temperature and relative humidity on elastic modulus of corrugated paper," Packaging Engineering, vol. 32, no. 21, pp. 45-48, 2011.

[12] Z. W. Wang and Y. P. E, "Mathematical modelling of energy absorption property for paper honeycomb in various ambient humidities," Materials and Corrosion, vol. 31, no. 9, pp. 43214328, 2010.

[13] Y. P. E and Z. W. Wang, "Effect of relative humidity on energy absorption properties of honeycomb paperboards," Packaging Technology and Science, vol. 23, no. 8, pp. 471-483, 2010.
[14] D. M. Wang, H. X. Gong, and Z. Y. Bai, "Effect investigation of relative humidity and temperature on multi-layer corrugated sandwich structures," Journal of Sandwich Structures and Materials, vol. 15, no. 2, pp. 156-167, 2013.

[15] Y. P. E and Z. W. Wang, "Energy-absorbing properties of paper honeycombs under low and intermediate strain rates," Packaging Technology and Science, vol. 25, no. 3, pp. 173-185, 2012.

[16] D. M. Wang and Z. Y. Bai, "Mechanical property of paper honeycomb structure under dynamic compression," Materials and Corrosion, vol. 77, pp. 59-64, 2015.

[17] Z. Y. Bai, D. M. Wang, and Z. F. Xu, "Model creation of strain rate-dependent energy absorption for paper honeycomb sandwich structure," Journal of Sandwich Structures and Materials, vol. 17, no. 4, pp. 359-375, 2015.

[18] D. M. Wang, J. Wang, and Q. H. Liao, "Investigation of mechanical property for paper honeycomb sandwich composite under different temperature and relative humidity," Journal of Reinforced Plastics and Composites, vol. 32, no. 13, pp. 987-997, 2013.

[19] W. Xiong, Energy absorption model research of paper honeycomb sandwich structure based on temperature and relative humidity [Master, thesis], Zhejiang Sci-Tech University, China, 2011.

[20] Z. F. Xu, Energy absorption model research of paper honeycomb sandwich structure based on strain rate [Master, thesis], Xian University of Technology, China, 2011. 


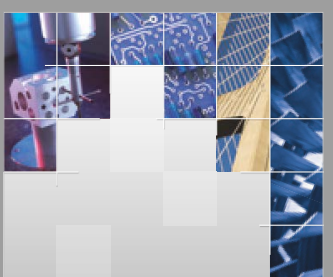

\section{Enfincering}
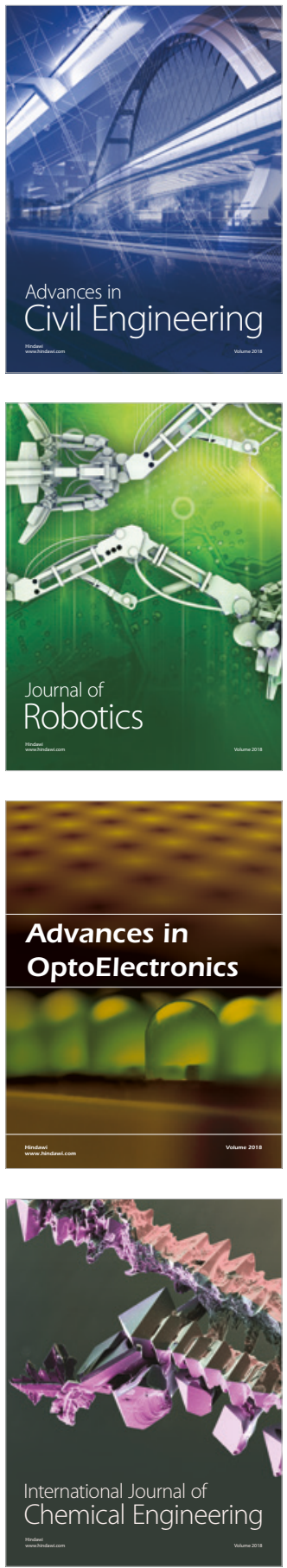

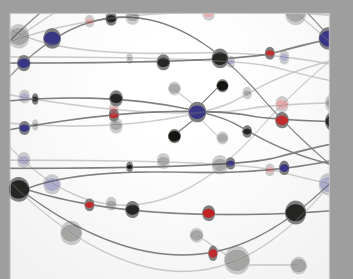

\section{Rotating \\ Machinery}

The Scientific World Journal

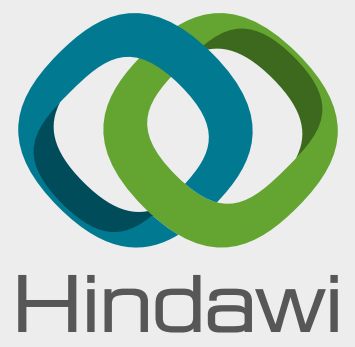

Submit your manuscripts at

www.hindawi.com
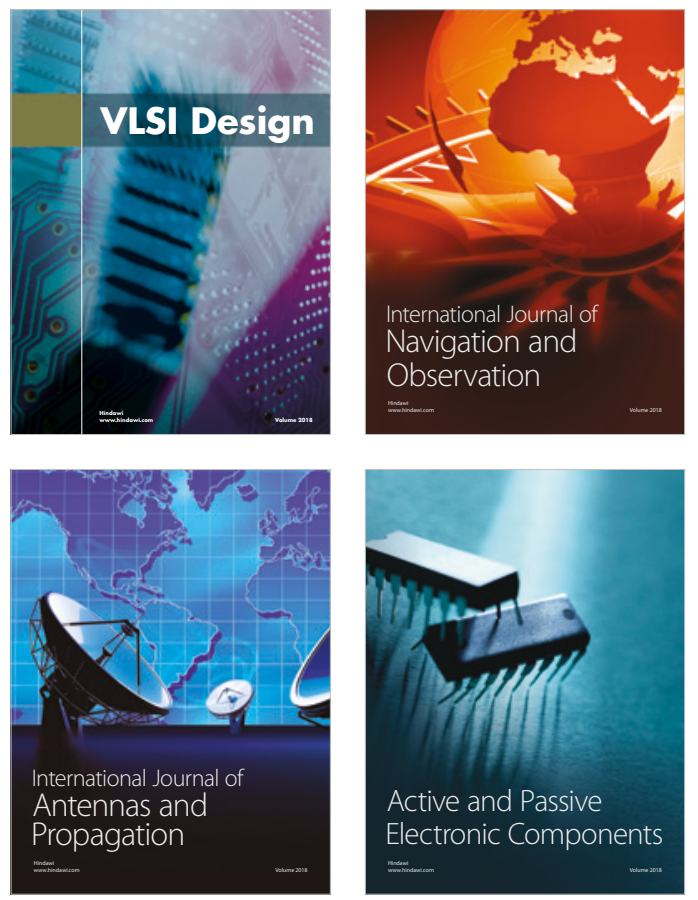
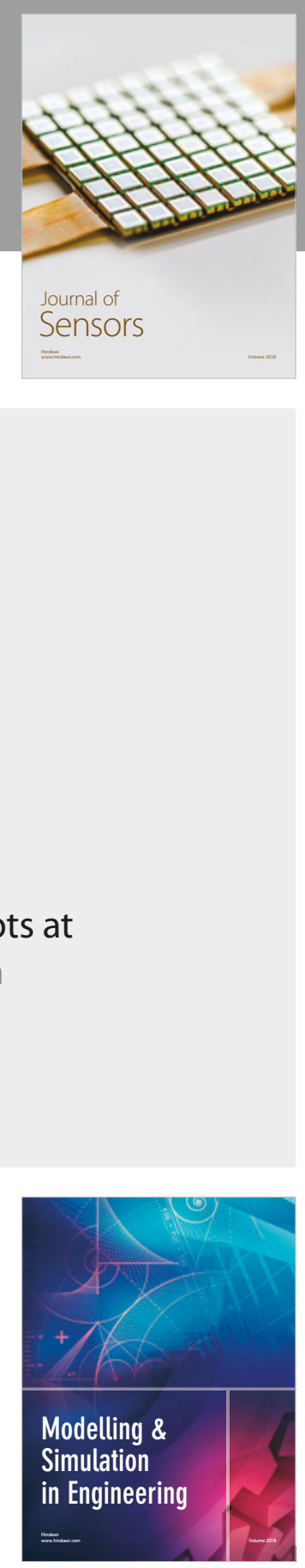

\section{Advances \\ Multimedia}
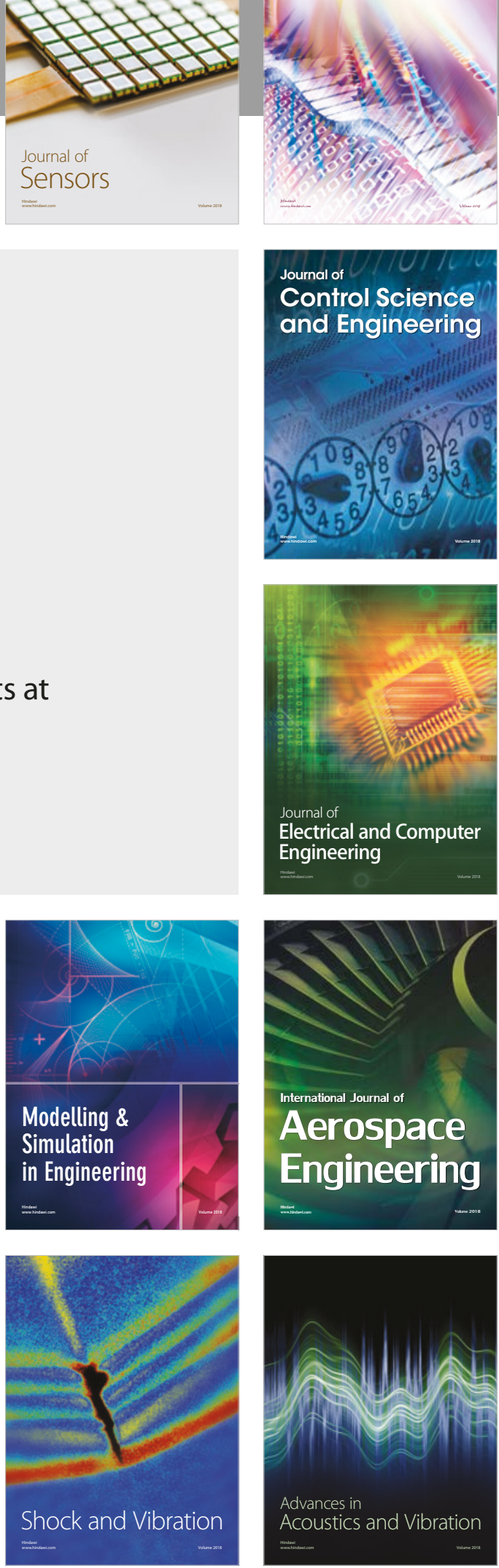